\title{
Osteotomía periacetabular de Ganz para el tratamiento de la displasia del desarrollo de la cadera: experiencia inicial y resultados de los primeros 44 casos
}

\author{
Gerardo Zanotti, Carlos M. Lucero, Fernando Díaz Dilernia, Pablo Slullitel, Fernando Comba, \\ Francisco Piccaluga, Martín Buttaro \\ Centro de Cadera "Sir John Charnley", Instituto de Ortopedia y Traumatología “Carlos E. Ottolenghi”, Hospital Italiano de Buenos Aires, \\ Ciudad Autónoma de Buenos Aires, Argentina
}

\begin{abstract}
RESUMEN
Objetivo: Describir la técnica quirúrgica, las indicaciones y los resultados iniciales de la osteotomía periacetabular bernesa para tratar la displasia del desarrollo de la cadera. Materiales y Métodos: Entre mayo de 2011 y mayo de 2020, se realizaron 44 osteotomías periacetabulares bernesas en 44 pacientes (35 mujeres, edad promedio 30 años [rango 23-38]). Todos tenían diagnóstico de displasia de cadera sintomática. El ángulo centro-borde promedio fue de $17^{\circ}$ (rango $9^{\circ}-20^{\circ}$ ) y el índice acetabular promedio, de $18^{\circ}$ (rango $15^{\circ}-20^{\circ}$ ). En 22 casos, se evaluaron y repararon los hallazgos intrarticulares por artroscopia en el mismo acto quirúrgico. Se evaluaron la corrección obtenida, la consolidación de la osteotomía y los resultados funcionales al final del seguimiento. Resultados: En 22 pacientes, se detectó hipertrofia y rotura del labrum acetabular asociadas a displasia de cadera. Diez pacientes tenían quistes paralabrales. El ángulo centro-borde promedio posoperatorio fue de $32^{\circ}\left(\right.$ rango $\left.27^{\circ}-35^{\circ}\right)$ y el índice acetabular, de $6^{\circ}$ (rango $4^{\circ}-9^{\circ}$ ). El tiempo quirúrgico para la osteotomía periacetabular bernesa fue de 130 min, cuando se sumó un procedimiento artroscópico, el tiempo fue de 148 minutos. Conclusiones: La osteotomía periacetabular bernesa es técnicamente demandante, pero logra resultados predecibles en pacientes con integridad del cartílago articular y deformidades corregibles. La artroscopia antes de la osteotomía permite evaluar las condiciones del cartílago, diagnosticar y tratar lesiones intrarticulares asociadas con esta enfermedad y decidir si es necesaria la corrección del déficit de cobertura.
\end{abstract}

Palabras clave: Displasia; cadera; artrosis; labrum; osteotomía; Ganz.

Nivel de Evidencia: IV

Ganz Periacetabular Osteotomy for the Treatment of Developmental Dysplasia of the Hip: Initial Experience and Results From the First 44 Cases

\begin{abstract}
Objective: To describe the surgical technique, indications, and initial results of the Bernese periacetabular osteotomy (PAO) for the treatment of developmental dysplasia of the hip. Materials and Methods: Between May 2011 and May 2020, 44 PAOs were performed in 44 patients ( 35 women) with an average age of 30 years (23-38). All patients had a diagnosis of symptomatic hip dysplasia. The average center-edge angle was $17^{\circ}\left(9^{\circ}\right.$ to $\left.20^{\circ}\right)$ and the average acetabular index was $18^{\circ}\left(15^{\circ}\right.$ to $\left.20^{\circ}\right)$. In 22 cases, the intra-articular findings were evaluated and repaired by arthroscopy in the same surgical stage. The correction obtained, the consolidation of the osteotomy, and the functional outcomes at the end of the follow-up were evaluated. Results: Hypertrophy and rupture of the acetabular labrum associated with hip dysplasia were evidenced in 22 patients. Paralabral cysts were found in 10 patients in the series. The average postoperative center-edge angle was $32^{\circ}\left(27^{\circ}\right.$ to $\left.35^{\circ}\right)$ and the acetabular index was $6^{\circ}\left(4^{\circ}\right.$ to $\left.9^{\circ}\right)$. The surgical time for PAO was 130 minutes; in patients where an arthroscopic procedure was added, the time was 148 minutes. Conclusions: PAO is technically demanding, but has predictable outcomes in patients with articular cartilage integrity and correctable deformities. Arthroscopy before osteotomy allows assessing cartilage conditions, diagnosing and treating intra-articular lesions associated with this pathology, and deciding on the need to correct the soft tissue deficit.
\end{abstract}

Key words: Dysplasia; hip; osteoarthrosis; labrum; osteotomy; Ganz.

Level of Evidence: IV

Recibido el 6-11-2020. Aceptado luego de la evaluación el 24-2-2021 • Dr. GERARDO ZANOTTI • gerardozanotti@ gmail.com

Cómo citar este artículo: Zanotti G, Lucero CM, Díaz Dilernia F, Slullitel P, Comba F, Piccaluga F, Buttaro M. Osteotomía periacetabular de Ganz para el tratamiento de la displasia del

desarrollo de la cadera: experiencia inicial y resultados de los primeros 44 casos. Rev Asoc Argent Ortop Traumatol 2021;86(6):727-736. https://doi.org/10.15417/issn. 1852-7434.2021.86.6.1286 


\section{INTRODUCCIÓN}

Las alteraciones anatómicas de la cadera conducen al desarrollo de daños articulares irreversibles. ${ }^{1,2}$ En la actualidad, la displasia del desarrollo de la cadera (DDC) y el síndrome de fricción femoroacetabular se consideran los cuadros que más comúnmente conducen a la artrosis de cadera en el adulto joven.

La osteotomía periacetabular bernesa (OPAB), descrita por Ganz es el tratamiento de elección para la DDC con retroversión acetabular o sin este procedimiento, ya que busca corregir la pobre cobertura o la incongruencia acetabular y, por ende, la inestabilidad que caracteriza a dicha enfermedad. ${ }^{4}$ El proceso de corrección se caracteriza por devolverle cobertura a la cabeza femoral, y corregir la anteversión y retroversión del acetábulo, según cada paciente en particular.

El paciente candidato a una OPAB es aquel que presenta una DDC sintomática, adolescente o adulto joven con deformidad corregible y rango de movilidad conservado. ${ }^{5}$ En general, está recomendada para pacientes ( $>10$ años) con cartílago trirradiado cerrado y pacientes $<40$ años debido a las características articulares más cercanas a la artrosis. Para los pacientes sintomáticos $>40$ años que tienen una cadera displásica, las indicaciones para ejecutar un procedimiento de preservación de la articulación son controvertidas. ${ }^{6-9}$

Las contraindicaciones para la OPAB incluyen artrosis avanzada (Tönnis 2 o 3), con subluxación articular que puede alterar la congruencia articular. Por otro lado, los pacientes con restricciones severas en el rango de movilidad también son malos candidatos para este procedimiento. ${ }^{10,11}$

El objetivo de este estudio es describir la técnica quirúrgica, resaltar las indicaciones e informar los resultados iniciales de una serie de 44 pacientes sometidos a una OPAB para tratar la DDC, en nuestro Centro. En segundo lugar, buscamos analizar los resultados clínicos y radiográficos a corto plazo de la osteotomía combinada con una artroscopia de cadera en el mismo acto quirúrgico.

\section{MATERIALES Y MÉTODOS}

Luego de obtener la aprobación del Comité de Ética de la institución, evaluamos retrospectivamente a 44 pacientes con DDC operados entre 2011 y 2020. En 22 casos, se había realizado una artroscopia de cadera, debido a una lesión labral, sin lesión de tipo CAM en el cuello femoral y se continuó con una OPAB en un mismo tiempo quirúrgico (grupo A); en los 22 casos restantes, se había efectuado una OPAB en forma aislada (grupo B), ya que no tenían una lesión del labrum ni lesión de tipo CAM en los estudios previos.

La información fue recolectada de la historia clínica electrónica de la institución, que está digitalizada desde 2004. La mediana de edad de la serie era de 30 años (rango intercuartílico [RIC] 23-38), 31 años (RIC 26-42) para el grupo A y 30 años (RIC 23-35) para el grupo B ( $\mathrm{p}=0,30)$. Quince pacientes del grupo A y 20 del grupo $B$ eran mujeres $(p=0,06)$. La mediana de seguimiento era de 28 meses (RIC 12-40), 34 meses (RIC 21-45) para el grupo A y 15 meses (RIC 11-30) para el grupo B $(\mathrm{p}=0,03)$. No se registraron pérdidas en el seguimiento (Tabla 1).

La evaluación radiográfica preoperatoria incluyó una incidencia de pelvis anteroposterior que permitió clasificar a los pacientes y determinar los ángulos acetabulares, y una de perfil del lado afectado con medición del ángulo alfa. Todos los casos correspondían al grado I de la clasificación de Crowe, ${ }^{12}$ porque tenían un desplazamiento proximal $<10 \%$ y una subluxación de la cabeza femoral $<50 \%$. La mediana del índice acetabular (IA) preoperatorio era de $19^{\circ}$ (RIC 16-20) para el grupo A y $17^{\circ}$ (RIC 15-18) para el grupo $\mathrm{B}(\mathrm{p}=0,14)$. La mediana del ángulo centro-borde lateral (ACBL) preoperatorio era de $16^{\circ}$ (RIC 8-19) para el grupo A y $19^{\circ}$ (RIC 10-20) para el otro grupo $(\mathrm{p}=0,19)$. Solo en siete y seis pacientes del grupo A y B, respectivamente, se observó una retroversión acetabular verdadera, definida por la presencia de dos de los siguientes signos radiográficos: signo del entrecruzamiento, signo de la pared posterior y signo de la espina ciática $(p=0,74)$ (Figura 1). En ambos grupos, se evaluó el compromiso articular utilizando la clasificación de Tönnis. ${ }^{13}$ El deterioro articular fue similar en ambos grupos $\mathrm{y}$, en ningún caso, fue superior al grado $2(\mathrm{p}=0,91)$ (Tabla 1 ).

La evaluación clínico-funcional se realizó antes de la cirugía y después con el Harris Hip Score modificado (HHSm), el puntaje de la University of California at Los Angeles (UCLA) y la escala analógica visual (EAV) para dolor. La mediana del HHSm preoperatorio fue de 61 (RIC 58-64) para el grupo A y 61 (RIC 57-61) para el grupo $\mathrm{B}(\mathrm{p}=0,39)$. La mediana del puntaje de la UCLA preoperatorio fue de 7 (RIC 6-8) para el primer grupo y 6 (RIC 6-8) para el segundo $(\mathrm{p}=0,42)$. El puntaje de la EAV preoperatorio tuvo una mediana de 8 (RIC 7-9) en el grupo A y 8 (RIC 7-8) en el grupo $B(p=0,37)$ (Tabla 1$)$. 


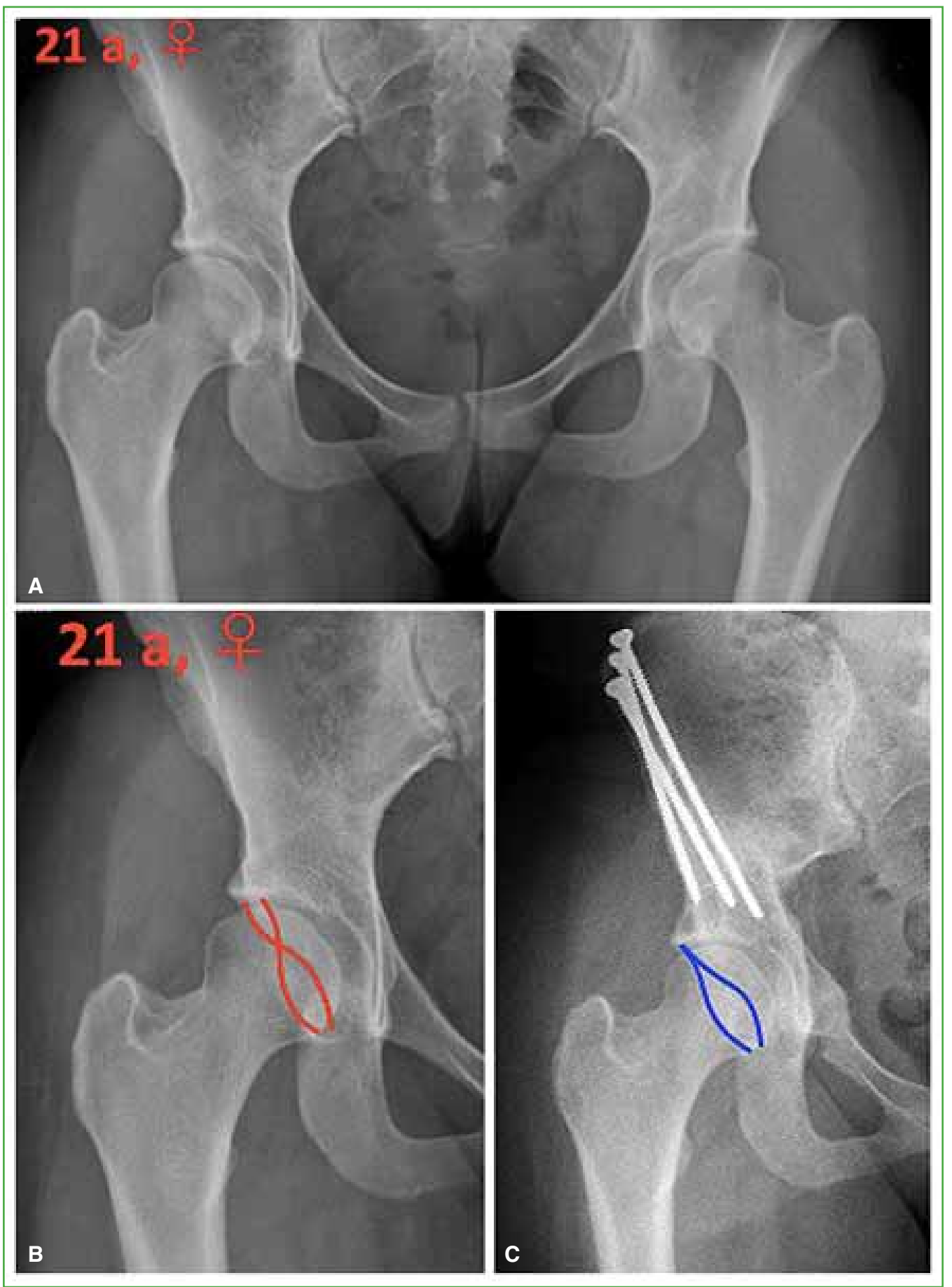

Figura 1. A. Radiografía anteroposterior de cadera preoperatoria de una paciente de 21 años con displasia del desarrollo de la cadera derecha sintomática. B. Imagen preoperatoria que muestra el signo del entrecruzamiento de ambas paredes del acetábulo. C. Imagen posoperatoria en la que se observa la osteosíntesis de la osteotomía periacetabular bernesa; nótese el descruzamiento de las paredes anterior y posterior del acetábulo. 
Tabla 1. Características demográficas de la serie

\begin{tabular}{|c|c|c|c|c|}
\hline Variable & Serie $(n=44)$ & Grupo A $(n=22)$ & Grupo B $(\mathrm{n}=22)$ & $\mathbf{p}$ \\
\hline Mediana de edad, años & 30 (RIC 23-38) & 31 (RIC 26-42) & 30 (RIC 23-35) & 0,30 \\
\hline Sexo, femenino/masculino & $35 / 9$ & $15 / 7$ & $20 / 2$ & 0,06 \\
\hline $\begin{array}{l}\text { Clasificación de la ASA, n (\%) } \\
1 \\
2\end{array}$ & $\begin{array}{l}24(55 \%) \\
20(45 \%)\end{array}$ & $\begin{array}{l}11(50 \%) \\
11(50 \%)\end{array}$ & $\begin{array}{r}13(59 \%) \\
9(41 \%)\end{array}$ & 0,44 \\
\hline $\begin{array}{l}\text { Clasificación de Tönnis, n (\%) } \\
0 \\
1 \\
2 \\
3\end{array}$ & $\begin{array}{c}19(43 \%) \\
18(41 \%) \\
7(16 \%) \\
-\end{array}$ & $\begin{array}{l}9(41 \%) \\
9(41 \%) \\
4(18 \%) \\
-\end{array}$ & $\begin{array}{c}10(45 \%) \\
9(41 \%) \\
3(14 \%) \\
-\end{array}$ & 0,91 \\
\hline $\begin{array}{l}\text { Retroversión acetabular, n (\%) } \\
\text { Sí } \\
\text { No }\end{array}$ & $\begin{array}{l}13(30 \%) \\
31(70 \%)\end{array}$ & $\begin{array}{c}7(32 \%) \\
15(68 \%)\end{array}$ & $\begin{array}{c}6(27 \%) \\
16(73 \%)\end{array}$ & 0,74 \\
\hline ACBL preoperatorio $\left(^{\circ}\right)$ & 17 (RIC 9-20) & 16 (RIC 8-19) & 19 (RIC 10-20) & 0,19 \\
\hline IA preoperatorio $\left({ }^{\circ}\right)$ & 18 (RIC 15-20) & 19 (RIC 16-20) & 17 (RIC 15-18) & 0,14 \\
\hline UCLA preoperatorio & 7 (RIC 6-8) & 7 (RIC 6-8) & 6 (RIC 6-8) & 0,42 \\
\hline HHSm preoperatorio & 61 (RIC 57-64) & 61 (RIC 58-64) & 61 (RIC 57-61) & 0,39 \\
\hline EAV preoperatorio & 8 (RIC 7-8) & 8 (RIC 7-9) & 8 (RIC 7-8) & 0,37 \\
\hline Tiempo quirúrgico (min) & 141 (RIC 129-175) & 148 (RIC 139-190) & 130 (RIC 113-151) & 0,004 \\
\hline Seguimiento (meses) & 28 (RIC 12-40) & 34 (RIC 21-45) & 15 (RIC 11-30) & 0,03 \\
\hline
\end{tabular}

RIC = rango intercuartílico, ASA = American Society of Anesthesiologists, ACBL = ángulo centro-borde lateral, IA = índice acetabular, UCLA = University of California at Los Angeles, HHSm = Harris Hip Score modificado, EAV = escala analógica visual.

Todas las cirugías estuvieron a cargo del mismo cirujano, especializado en patología de la cadera siguiendo los principios descritos para ambas técnicas quirúrgicas. ${ }^{14,15}$ El cirujano senior realizó un fellowship en cirugía de preservación articular en un centro de alto volumen con un nivel de responsabilidad creciente durante seis meses. Ninguno de los pacientes había sido operado antes. Se utilizó la clasificación de la American Society of Anesthesiologists (ASA) ${ }^{16}$ para categorizar el estado fisiológico preoperatorio: 11 pacientes del grupo A y 13 del grupo B eran ASA I $(\mathrm{p}=0,44)$. Bajo anestesia epidural hipotensiva, se realizó el procedimiento artroscópico en un primer tiempo quirúrgico, sobre una mesa de tracción, en los pacientes del grupo A. Luego del cambio a una mesa radiolúcida y la colocación de nuevos campos estériles, se efectuó un abordaje de Smith-Petersen en todos los pacientes para continuar con la OPAB. En el grupo A, la reparación labral artroscópica se efectuó con arpones biodegradables de 3,2 mm (Arthrex ${ }^{\circledR}$ ). La cantidad de arpones utilizados dependió de los que fueran necesarios para obtener la estabilidad labral en cada caso específico, sin contar con un protocolo previo. No se reparó la capsulotomía al finalizar el procedimiento artroscópico en ningún paciente. Luego de obtener la corrección acetabular deseada con la OPAB, el fragmento se fijó con tornillos de cortical de 4,5 $\mathrm{mm}$ en ambos grupos (DePuy-Synthes ${ }^{\circledR}$ ) (Figura 2). En ningún paciente del grupo $B$, se realizó la reparación labral. La mediana del tiempo quirúrgico de la serie fue de 141 min (RIC 129-175), 148 min (RIC 139-190) para el grupo A y 130 min (RIC 113-151) para el grupo B $(\mathrm{p}=0,004)$. 


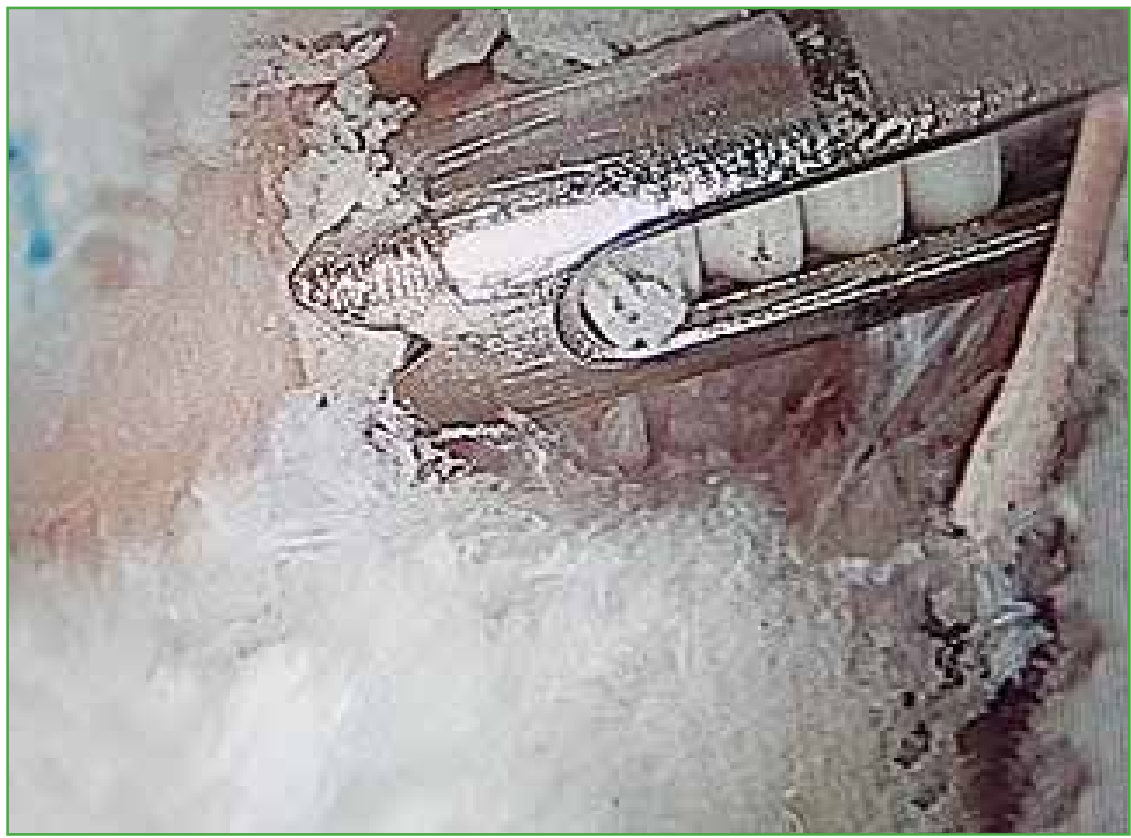

Figura 2. Imagen clínica intraoperatoria. Se puede observar la colocación de un arpón de $3,2 \mathrm{~mm}$ en el procedimiento artroscópico, durante el primer tiempo quirúrgico.

Se administró profilaxis antitrombótica con heparina de bajo peso molecular (40 mg/día) por vía subcutánea, durante cuatro semanas a los pacientes de ambos grupos. ${ }^{17}$ No se indicó un tratamiento profiláctico de rutina para las calcificaciones heterotópicas. Luego de la cirugía, el miembro inferior operado fue colocado en posición neutra. Debido a la preservación de la continuidad del anillo pélvico, se autorizó la deambulación precoz con asistencia. Se indicó una carga parcial de $15 \mathrm{~kg}$ de peso sobre el lado operado durante las primeras ocho semanas, realizando ejercicios de movilidad de la cadera con flexión limitada a $90^{\circ}$, rotación interna neutra, $30^{\circ}$ de rotación externa y $30^{\circ}$ de abducción por 3-6 semanas. Los movimientos activos y pasivos continuos fueron supervisados por el Servicio de Kinesiología desde el primer día posterior a la cirugía para prevenir las adherencias capsulares. Se permitió el aumento gradual de la carga de peso cuando se observaron signos de consolidación ósea en el control radiográfico posoperatorio de las ocho semanas. Luego continuaron con fisiokinesioterapia en los siguientes 2-3 meses para mejorar el rango de movilidad y la fuerza muscular. El seguimiento clínico y radiográfico se realizó a los 15 y 45 días, y a los 3, 6 y 12 meses de la cirugía y luego anualmente, con una radiografía anteroposterior, oblicuas alar y obturatriz, y de perfil de cadera del lado afectado. Se consideró que la OPAB estaba consolidada cuando se observaban puentes óseos en dos de las incidencias radiográficas. ${ }^{18}$ Se registraron las complicaciones y se consideró falla infecciosa a todo caso que requirió un tratamiento quirúrgico adicional por infección del sitio quirúrgico; y falla no infecciosa a todo paciente que requirió una cirugía de revisión, sin importar la causa.

\section{Análisis estadístico}

Las variables continuas se expresan como mediana y RIC o como promedio y desviación estándar (DE), según si tienen una distribución anormal o no, respectivamente. Las variables categóricas se expresan como frecuencias y porcentajes. Las variables continuas fueron comparadas utilizando la prueba t de Student cuando tuvieron una distribución normal y la prueba U de Mann-Whitney cuando no la tuvieron. Se emplearon la prueba de ji al cuadrado y exacta de Fisher para comparar variables categóricas. Se compararon las variables demográficas y los hallazgos radiográficos de ambos grupos para analizar la evolución de los resultados clínicos. Se realizó un análisis de regresión logística multivariada para evaluar si el procedimiento artroscópico adicional se asocia a una mejoría en los resultados clínico-funcionales posoperatorios. Se consideró estadísticamente significativo un valor p <0,05. Se empleó el programa STATA 13 ${ }^{\mathrm{TM}}$ (Stata Corp., College Station, Texas, EE.UU.) para el análisis estadístico. 


\section{RESULTADOS}

Análisis radiográfico

No se registraron casos de progresión de la degeneración articular en ninguno de los dos grupos. Con respecto a las mediciones angulares posoperatorias, la mediana del IA mejoró en un valor de $11^{\circ}$ (RIC 9-13) y el ACBL en un valor de $16^{\circ}$ (RIC 14-18) en toda la serie $(\mathrm{p}<0,001)$, pero no se registró una diferencia estadísticamente significativa en la medición posoperatoria entre ambos grupos (Tabla 2).

Tabla 2. Resultados posoperatorios y complicaciones por grupo

\begin{tabular}{|c|c|c|c|c|}
\hline Variable & Serie $(n=44)$ & Grupo A $(\mathrm{n}=22)$ & Grupo B (n =22) & p \\
\hline ACBL posoperatorio $\left({ }^{\circ}\right)$ & 32 (RIC 27-35) & 32 (RIC 26-35) & 32 (RIC 30-35) & 0,53 \\
\hline IA posoperatorio $\left({ }^{\circ}\right)$ & 6 (RIC 4-9) & 7 (RIC 6-11) & 5 (RIC 4-8) & 0,12 \\
\hline Mejoría del ACBL posoperatorio $\left(^{\circ}\right)$ & 16 (RIC 14-18) & 16 (RIC 14-22) & 16 (RIC 13-16) & 0,47 \\
\hline Mejoría del IA posoperatorio $\left(^{\circ}\right)$ & 11 (RIC 9-13) & 9 (RIC 8-12) & 11 (RIC 10-13) & 0,28 \\
\hline UCLA posoperatorio & 8 (RIC 7-8) & 7 (RIC 6-8) & 8 (RIC 7-8) & 0,33 \\
\hline HHSm posoperatorio & 87 (RIC 87-91) & 87 (RIC 87-91) & 87 (RIC 87-87) & 0,15 \\
\hline EAV posoperatoria & $1(\mathrm{RIC} 0-1)$ & 0 (RIC 0-2) & $1(\mathrm{RIC} 0-1)$ & 0,84 \\
\hline Mejoría UCLA posoperatorio & $1(\mathrm{RIC} 0-2)$ & $1(\mathrm{RIC} 0-1)$ & $1(\mathrm{RIC} 0-2)$ & 0,18 \\
\hline Mejoría HHSm posoperatorio & 26 (RIC 26-30) & 27 (RIC 25-29) & 26 (RIC 26-30) & 0,84 \\
\hline Mejoría EAV posoperatoria & 7 (RIC 6-8) & 7 (RIC 5-8) & 7 (RIC 6-7) & 0,50 \\
\hline $\begin{array}{l}\text { Complicaciones } \\
\text { Fractura pared posterior } \\
\text { Compresión NFC } \\
\text { Infección superficial }\end{array}$ & $\begin{array}{c}5(11 \%) \\
1 \\
2 \\
2\end{array}$ & $\begin{array}{c}2(9 \%) \\
1 \\
1\end{array}$ & $\begin{array}{c}3(14 \%) \\
1 \\
2\end{array}$ & 0,64 \\
\hline \multicolumn{5}{|c|}{ Resultados posoperatorios generales de la serie } \\
\hline Variable & Serie $(n=44)$ & & $\mathbf{p}$ & \\
\hline Mejoría del ACBL posoperatorio $\left({ }^{\circ}\right)$ & 16 (RIC 14-18) & & $<0,001$ & \\
\hline Mejoría del IA posoperatorio $\left({ }^{\circ}\right)$ & $11(\mathrm{RIC} 9-13)$ & & $<0,001$ & \\
\hline Mejoría UCLA posoperatorio & $1(\mathrm{RIC} 0-2)$ & & $<0,001$ & \\
\hline Mejoría HHSm posoperatorio & 26 (RIC 26-30) & & $<0,001$ & \\
\hline Mejoría EAV posoperatoria & 7 (RIC 6-8) & & $<0,001$ & \\
\hline
\end{tabular}

$\mathrm{RIC}=$ rango intercuartílico, $\mathrm{ACBL}=$ ángulo centro-borde lateral, $\mathrm{IA}=$ índice acetabular, UCLA = University of California at Los Angeles, HHSm $=$ Harris Hip Score modificado, EAV = escala analógica visual, NFC = nervio femorocutáneo. 


\section{Evaluación funcional}

La mediana del HHSm posoperatorio fue de 87 (RIC 87-91) para el grupo A y 87 (RIC 87-87) para el grupo B $(\mathrm{p}=0,15)$. La mediana del puntaje de la UCLA posoperatorio fue de 7 (RIC 6-8) para el primer grupo y 8 (RIC 7-8) para el segundo $(\mathrm{p}=0,33)$. En la EAV del posoperatorio, los pacientes del grupo A manifestaron una mediana de dolor de 0 (RIC 0-2) y los del grupo B, una mediana de 1 (RIC 0-1) ( $\mathrm{p}=0,84)$. Solo una paciente del grupo A desarrolló un síndrome de dolor regional complejo y mantuvo un valor igual al del preoperatorio (puntaje 7). En el análisis general de la serie, la mejoría posoperatoria de los puntajes del HHSm y de la UCLA fue de 26 (RIC 26-30) y de 1 (RIC 0-2), respectivamente, ( $\mathrm{p}<0,001$ ). El dolor preoperatorio mejoró 7 puntos en promedio (RIC 6-8) en la EAV ( $\mathrm{p}<0,001)$. No se registraron diferencias significativas en la medición de la mejoría posoperatoria, entre ambos grupos (Tabla 2).

\section{Complicaciones}

No se registraron complicaciones neurovasculares mayores en ningún grupo. Hubo dos complicaciones en el grupo A: un paciente sufrió una fractura incompleta sin desplazamiento de la pared posterior del acetábulo durante la cirugía que no influyó en la rehabilitación posterior, y otro refirió una meralgia parestésica por el compromiso del nervio femorocutáneo, que mejoró notablemente al cabo de 90 días y no requirió intervención posterior. En el grupo B, también se registró un compromiso sensitivo del nervio femorocutáneo que se resolvió con tratamiento conservador al igual que en el paciente del grupo A. Por último, dos pacientes sufrieron una infección superficial de la herida quirúrgica, que se curó con antibióticos por vía oral, durante 14 días [grupo A (1) vs. grupo B (1), p $=0,64]$ (Tabla 2).

\section{Análisis de regresión lineal}

El análisis de regresión lineal univariado no encontró asociación estadísticamente significativa entre ninguna de las variables de la evaluación clínico-funcional con respecto a la realización del procedimiento artroscópico (Tabla 3).

Tabla 3. Modelo de regresión lineal univariable con el procedimiento artroscópico como variable dependiente

\begin{tabular}{|l|c|c|}
\hline \multirow{2}{*}{ Variable } & \multicolumn{2}{|c|}{ Análisis univariable } \\
\cline { 2 - 3 } & B - coeficiente (IC95\%) & p \\
\hline Mejoría UCLA posoperatorio & $-0,06(-0,15-0,02)$ & 0,14 \\
\hline Mejoría HHSm posoperatorio & $-0,01(-0,03-0,03)$ & 0,84 \\
\hline Mejoría EAV posoperatoria & $0,01(-0,07-0,08)$ & 0,83
\end{tabular}

UCLA = University of California at Los Angeles, HHSm = Harris Hip Score modificado, EAV = escala analógica visual.

\section{DISCUSIÓN}

La osteotomía de Ganz está ampliamente reconocida como la más anatómica de las osteotomías de redirección periacetabular; no obstante, es el procedimiento más complejo, pero ha logrado un éxito reproducible a corto plazo.

Es fundamental ser estrictos en cada paso de la cirugía. Para esto se debe controlar bajo fluoroscopio cada gesto que se realice durante el procedimiento. Se debe ser muy cuidadoso al ejecutar la primera osteotomía, ya que un corte muy bajo dificulta la correcta posición de los fragmentos, y si es muy alto, se puede vulnerar la articulación, una de las complicaciones más temidas (Figura 3). El tercer corte es, a nuestro entender, el más importante, porque transcurre entre la columna posterior, a medial, y la articulación, a lateral. Cualquier variación en este corte puede llevar a la violación de la articulación, vulnerar la columna posterior y, por ende, comprometer la estabilidad propia de la pelvis. El corte en el hueso ilíaco es otro punto importante por tener en cuenta. Es fundamental respetar la distancia del corte a la superficie superior del acetábulo, ya que las osteotomías muy cerca pueden dañar la arteria glútea media y obturatriz generando una necrosis acetabular. ${ }^{19}$ 


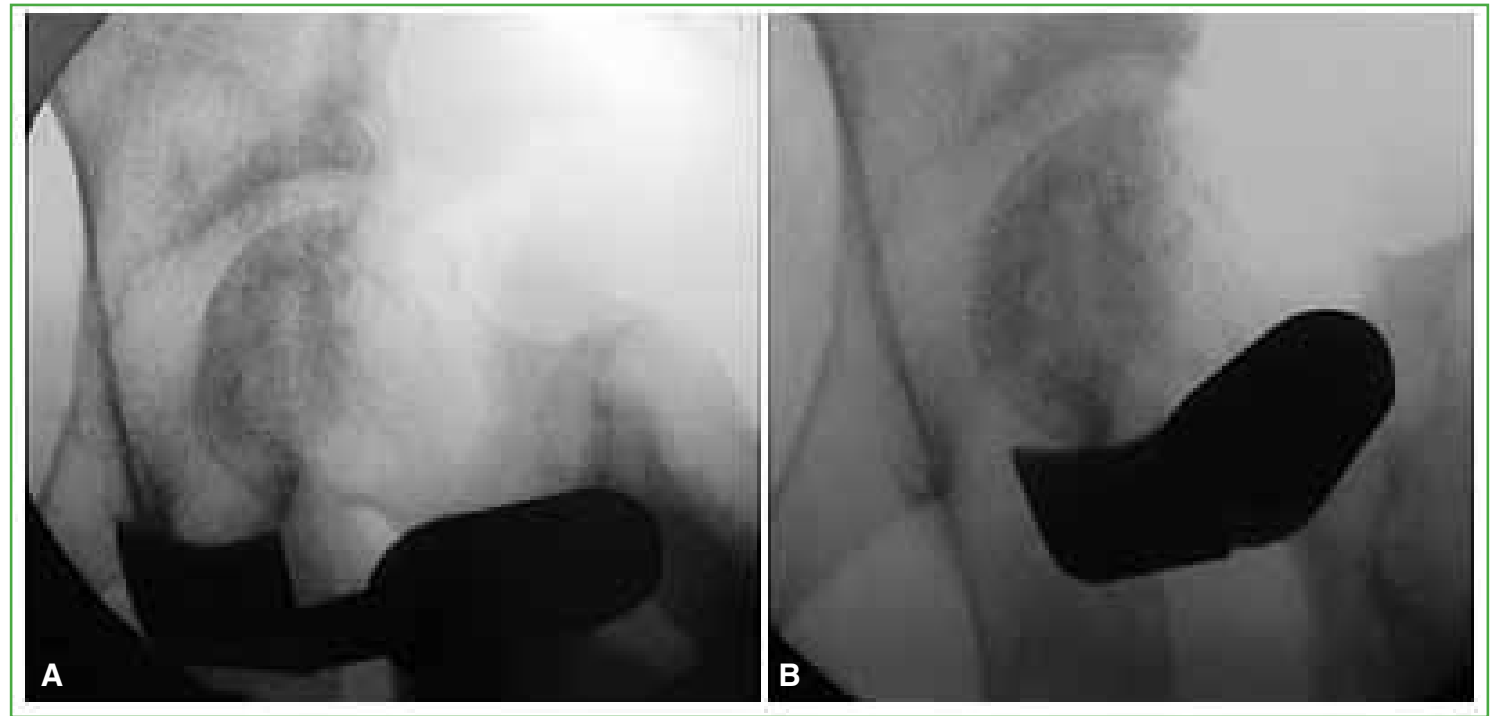

Figura 3. Imágenes intraoperatorias con intensificador. Se observa la osteotomía en el isquion a nivel medial (A) y lateral (B).

Es necesario y fundamental el control intraquirúrgico de la posición final de la corrección del acetábulo. Antes de comenzar la cirugía, es obligatorio realizar un control radiográfico de la posición de la pelvis sobre la mesa quirúrgica respetando los parámetros que describió Tannast. ${ }^{20}$ Una vez realizada la corrección acetabular, se toma una proyección anteroposterior de la cadera para evaluar la correcta posición acetabular. En esta proyección, se evalúan la cobertura de la cabeza femoral midiendo los ángulos ACBL y el IA, la posición de las paredes anterior y posterior del acetábulo, la distancia entre la cabeza femoral y el fondo del acetábulo, el arco de Shenton y la posición de la imagen en lágrima.

En 22 pacientes, se realizó una artroscopia de cadera antes de la OPAB, en el mismo acto quirúrgico. Este procedimiento es beneficioso por dos motivos. Primero, le permite al cirujano identificar la patología intrarticular. Los desgarros del labrum, los colgajos condrales y la condromalacia pueden observarse directamente. Esto puede tener un valor pronóstico importante para los pacientes que se someten a una OPAB y, en algunas circunstancias, puede llevar al cirujano a tomar la decisión de no continuar con una osteotomía inmediatamente después del procedimiento artroscópico. En todos los pacientes, se constató una lesión del labrum y se reparó con arpones. La cantidad de anclajes utilizados dependió de los que fueran necesarios en cada caso específico para obtener la estabilidad labral, sin contar con un protocolo previo.

En cuanto a la evaluación radiográfica de las mediciones angulares posoperatorias, la mediana del IA mejoró en $11^{\circ}$ (RIC 9-15) y el ACBL en $18^{\circ}$ (RIC $\left.15-24\right)$ en toda la serie $(\mathrm{p}<0,001)$, pero no se registró una diferencia significativa en la medición posoperatoria entre ambos grupos (Tabla 2). El ACBL posoperatorio promedio fue de $32^{\circ}$ en la serie ( $\operatorname{RIC} 27^{\circ}-35^{\circ}$ ): grupo A $32^{\circ}\left(\right.$ RIC $26^{\circ}-35^{\circ}$ ) y grupo B: $32\left(\right.$ RIC $\left.30^{\circ}-35^{\circ}\right)$. El IA posoperatorio fue de $6^{\circ}\left(\mathrm{RIC} 4^{\circ}-9^{\circ}\right)$ : grupo A $7^{\circ}\left(\operatorname{RIC~}^{\circ}-11^{\circ}\right)$ y grupo B $5^{\circ}\left(\right.$ RIC $\left.4^{\circ}-8^{\circ}\right)$. Estas mediciones se mantuvieron en el tiempo, sin sufrir cambios en los controles posoperatorios.

Existe un período de curva de aprendizaje bien informado para la OPAB, que se cree que incluye aproximadamente las primeras 20 osteotomías y durante el cual las complicaciones quirúrgicas mayores no son infrecuentes. De acuerdo con nuestra experiencia inicial, la tasa de complicaciones en los primeros 44 casos operados en nuestra institución fue del $11 \%$.

En ningún paciente de nuestra serie, fue necesaria la conversión a prótesis total de cadera. Si bien el seguimiento es corto, existen series que muestran malos resultados en pacientes $>40$ años al operarse, como un menor puntaje preoperatorio de Merle d'Aubigné y Postel, una prueba de pinzamiento anterior preoperatoria positiva, cojera preoperatoria y puntaje preoperatorio aumentado de artrosis según la clasificación de Tönnis. ${ }^{21-23}$ En la bibliografía, se sugiere firmemente que los pacientes jóvenes con caderas displásicas y cambios articulares mínimos o leves son los candidatos ideales para esta operación. En aquellos pacientes con cambios articulares moderados o severos, la OPAB se vuelve menos predecible y la tasa de conversión a una prótesis total de cadera es alta. ${ }^{24-26}$ 
Nuestro estudio presenta varias limitaciones. En primer lugar, existe el sesgo de selección debido a su naturaleza retrospectiva. Segundo, los datos inherentes al rango de movilidad fueron registrados por diferentes autores, sin una prueba de confiabilidad entre los medidores, por lo que existe el riesgo de error de medición y sesgo de evaluación. Por otro lado, decidimos utilizar el HHSm como un resultado clínico basado en su validez y confiabilidad, pero tiene la limitación de que no evalúa la función durante las actividades deportivas y recreativas ni la calidad de vida relacionada con la cadera. Sin embargo, nuestro estudio compara un grupo de pacientes en quienes se realizó la OPAB solo frente a un grupo sometido a una artroscopia de cadera antes de la osteotomía, y se pudo observar una íntima relación entre el tratamiento combinado de manera secuencial. Además, nuestro estudio evidencia datos sólidos en el seguimiento a corto plazo relacionados con los resultados funcionales y las complicaciones en un centro de alto volumen quirúrgico con cirujanos entrenados. Como fortaleza del estudio, remarcamos que todos los pacientes fueron operados por un mismo cirujano.

\section{CONCLUSIONES}

Los primeros resultados en este grupo inicial de pacientes tratados con OPAB en nuestra institución muestran una corrección radiográfica confiable de la deformidad y una mejora en la función, con una tasa de complicaciones aceptable. Creemos que la ejecución de una artroscopia de cadera antes de la OPAB en el mismo acto quirúrgico es una opción válida para evaluar el estado articular sin aumentar la morbilidad del procedimiento; sin embargo, no hemos podido demostrar una asociación estadísticamente significativa entre ninguna de las variables de la evaluación clínico-funcional con respecto a la realización del procedimiento artroscópico.

Conflicto de intereses: Los autores no declaran conflictos de intereses.

ORCID de C. M. Lucero: https://orcid.org/0000-0003-1325-7027 ORCID de F. Díaz Dilernia: https://orcid.org/0000-0002-7830-2207 ORCID de P. Slullitel: https://orcid.org/0000-0002-8957-075X
ORCID de F. Comba: https://orcid.org/0000-0002-2848-2983

ORCID de F. Piccaluga: https://orcid.org/0000-0002-9887-4886

ORCID de M. Buttaro: https://orcid.org/0000-0003-3329-778X

\section{BIBLIOGRAFÍA}

1. Murphy SB, Ganz R, Muller ME. The prognosis in untreated dysplasia of the hip: a study of radiographic factors that predict the outcome. J Bone Joint Surg Am 1995;77(7):985-9.

https://doi.org/10.2106/00004623-199507000-00002

2. Wilkin GP, Ibrahim MM, Smit KM, Beaulé PE. A contemporary definition of hip dysplasia and structural instability: toward a comprehensive classification for acetabular dysplasia. J Arthroplasty 2017;32(9S):S20-S27. https://doi.org/10.1016/j.arth.2017.02.067

3. Ganz R, Parvizi J, Beck M, Leunig M, Notzli H, Sibenrock KA. Femoroacetabular impingement: a cause for osteoarthritis of the hip. Clin Orthop Relat Res 2003(417):112-20. https://doi.org/10.1097/01.blo.0000096804.78689.c2

4. Ganz R, Klaue K, Vinh TS, Mast JW. The classic: A new periacetabular osteotomy for the treatment of hip dysplasias. Clin Orthop Relat Res 2004;(418):3-8. https://doi.org/10.1097/00003086-200401000-00002

5. Clohisy JC, Schutz AL, St John L, Schoenecker PL, Wright RW. Periacetabular osteotomy: a systematic literature review. Clin Orthop Relat Res 2009;467(8):2041-52. https://doi.org/10.1007/s11999-009-0842-6

6. Garbuz DS, Awwad MA, Duncan CP. Periacetabular osteotomy and total hip arthroplasty in patients older than 40 years. J Arthroplasty 2008;23(7):960-3. https://doi.org/10.1016/j.arth.2007.08.015

7. Hsieh PH, Huang KC, Lee PC, Chang YH. Comparison of periacetabular osteotomy and total hip replacement in the same patient: a two- to ten-year follow-up study. J Bone Joint Surg Br 2009;91(7):883-8. https://doi.org/10.1302/0301-620X.91B7.22183

8. Sharifi E, Sharifi H, Morshed S, Bozic K, Diab M. Cost-effectiveness analysis of periacetabular osteotomy. J Bone Joint Surg Am 2008;90(7):1447-56. https://doi.org/10.2106/JBJS.G.00730 
9. Teratani T, Naito M, Kiyama T, Maeyama A. Periacetabular osteotomy in patients fifty years of age or older. $J$ Bone Joint Surg Am 2010;92(1)31-41. https://doi.org/10.2106/JBJS.H.01556

10. Fujii M, Nakashima Y, Noguchi Y, Yamamoto T, Mawatari T, Motomura G, et al. Effect of intra-articular lesions on the outcome of periacetabular osteotomy in patients with symptomatic hip dysplasia. J Bone Joint Surg Br 2011;93(11):1449-56. https://doi.org/10.1302/0301-620X.93B11.27314

11. Yasunaga Y, Ikuta Y, Kanazawa T, Takahashi K, Hisatome T. The state of the articular cartilage at the time of surgery as an indication for rotational acetabular osteotomy. J Bone Joint Surg Br 2001;83(7):1001-4. https://doi.org/10.1302/0301-620x.83b7.12171

12. Jawad MU, Scully SP. In brief: Crowe's classification: arthroplasty in developmental dysplasia of the hip. Clin Orthop Relat Res 2011;469(1):306-8. https://doi.org/10.1007/s11999-010-1316-6

13. Kovalenko B, Bremjit P, Fernando N. Classifications in Brief: Tönnis classification of hip osteoarthritis. Clin Orthop Relat Res 2018;476(8):1680-4. https://doi.org/10.1097/01.blo.0000534679.75870.5f

14. Byrd JW, Pappas JN, Pedley MJ. Hip arthroscopy: an anatomic study of portal placement and relationship to the extra-articular structures. Arthroscopy 1995;11(4):418-23. https://doi.org/10.1016/0749-8063(95)90193-0

15. Leunig M, Ganz R. Berner periazetabuläre Osteotomie [The Bernese method of periacetabular osteotomy]. Orthopade 1998;27(11):743-50. https://doi.org/10.1007/pl00003460

16. Fitz-Henry J. The ASA classification and peri-operative risk. Ann R Coll Surg Engl 2011;93(3):185-7. https://doi.org/10.1308/rcsann.2011.93.3.185a

17. Flevas DA, Megaloikonomos PD, Dimopoulos L, Mitsiokapa E, Koulouvaris P, Mavrogenis AF. Thromboembolism prophylaxis in orthopaedics: an update. EFORT Open Rev 2018;3(4):136-48.

https://doi.org/10.1302/2058-5241.3.170018

18. Marsell R, Einhorn TA. The biology of fracture healing. Injury 2011;42(6):551-5. https://doi.org/10.1016/j.injury.2011.03.031

19. Beck M, Leunig M, Ellis T, Sledge JB, Ganz R. The acetabular blood supply: implications for periacetabular osteotomies. Surg Radiol Anat 2003;25(5-6):361-7. https://doi.org/10.1007/s00276-003-0149-3

20. Tannast M, Fritsch S, Zheng G, Siebenrock KA, Steppacher SD. Which radiographic hip parameters do not have to be corrected for pelvic rotation and tilt? Clin Orthop Relat Res 2015;473(4):1255-66. https://doi.org/10.1007/s11999-014-3936-8

21. Peters CL, Erickson JA, Hines JL. Early results of the Bernese periacetabular osteotomy: the learning curve at an academic medical center. J Bone Joint Surg Am 2006;88(9):1920-6. https://doi.org/10.2106/JBJS.E.00515

22. Pogliacomi F, Stark A, Wallensten R. Periacetabular osteotomy. Good pain relief in symptomatic hip dysplasia, 32 patients followed for 4 years. Acta Orthop 2005;76(1):67-74. https://doi.org/10.1080/00016470510030346

23. Steppacher SD, Tannast M, Ganz R, Siebenrock KA. Mean 20-year follow-up of Bernese periacetabular osteotomy. Clin Orthop Relat Res 2008;466(7):1633-44. https://doi.org/10.1007/s11999-008-0242-3

24. Trousdale RT, Cabanela ME. Lessons learned after more than 250 periacetabular osteotomies. Acta Orthop Scand 2003;74(2):119-26. https://doi.org/10.1080/00016470310013824

25. Okano K, Enomoto H, Osaki M, Shindo H. Joint congruency as an indication for rotational acetabular osteotomy. Clin Orthop Relat Res 2009;467:894-900. https://doi.org/10.1007/s11999-008-0443-9

26. Troelsen A, Elmengaard B, Soballe K. Medium-term outcome of periacetabular osteotomy and predictors of conversion to total hip replacement. J Bone Joint Surg Am 2009;91(9):2169-79.

https://doi.org/10.2106/JBJS.H.00994 